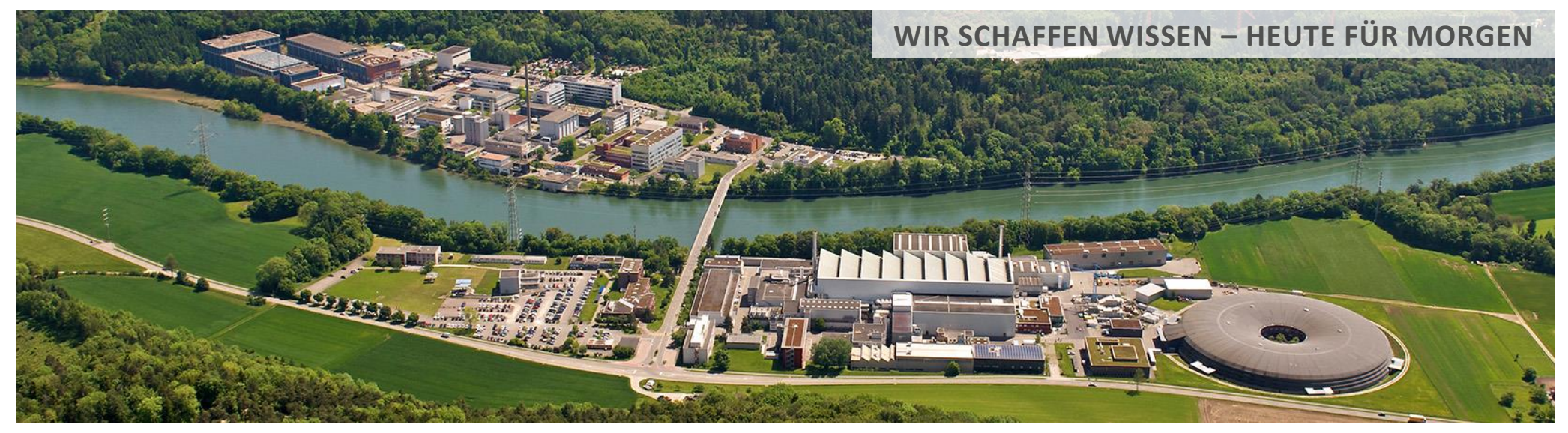

Dr. Nicola Casati :: Group Leader, Material Science :: Paul Scherrer Institut

\title{
High Throughput Powder Diffraction
}

The $2^{\text {nd }}$ International Online Conference on Crystals 
- Tunable beam size (ideal for complex chambers or small samples)

- Tunable wavelength (Energy)

- Short for more penetration, resolution

- Long for higher cross-section

- Move across elemental edges

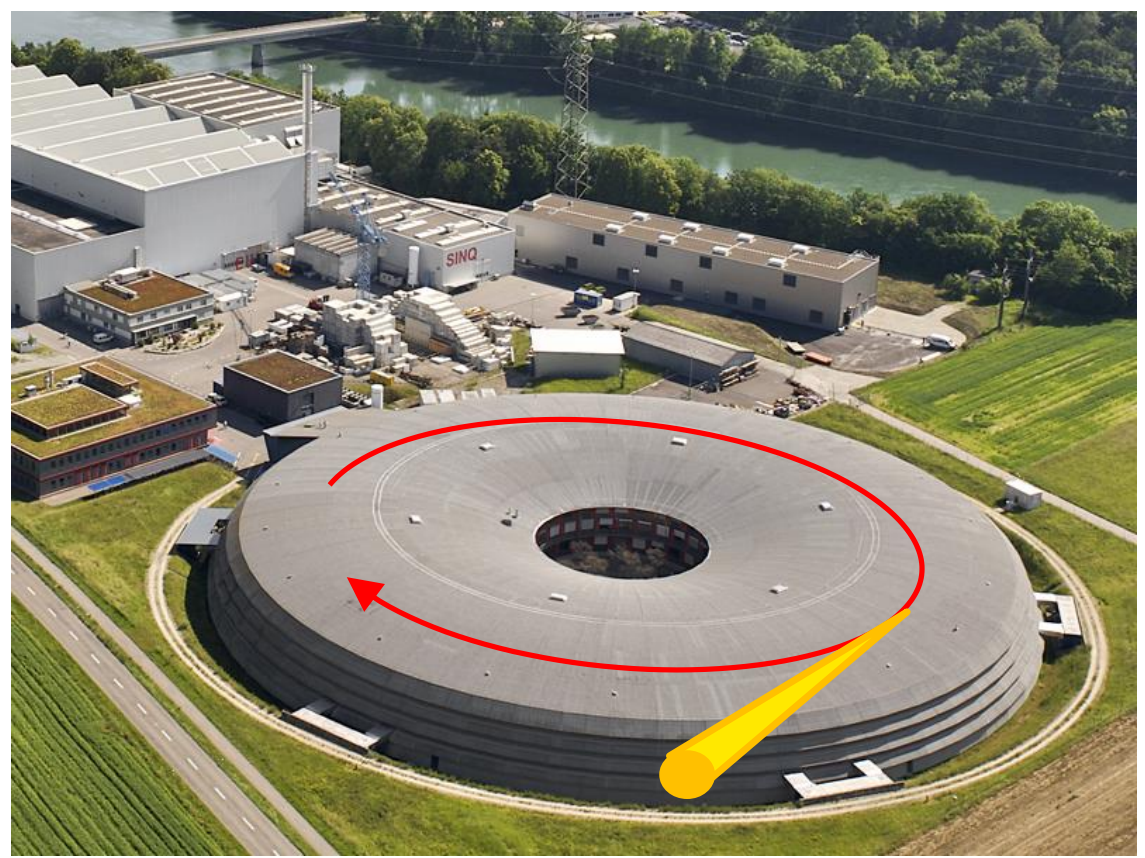

Synchrotrons are also correctly associated to the highly intense radiation beam they produce, which can be exploited in several different ways

- Better $\Delta \lambda / \lambda$ (to select a highly monochromatic radiation)

- Larger signal/noise ratio (quality)

- Time resolved (quantity, or time resolution within an experiment)

- High throughput (quantity, more samples within time)

We will focus today on the last point, which is often neglected as a 'brute force' approach 


\section{Transmission (Debye-Scherrer)}

In powder diffraction, the traditional acquisition method, by scanning the detector angularly around the sample, has been progressively overtaken by more complex ones.

Acquisition time

The single point-detector has progressively been

$\sim 10^{3}$ seconds substituted with several parallel ones, improving manifold the acquisition strategy.
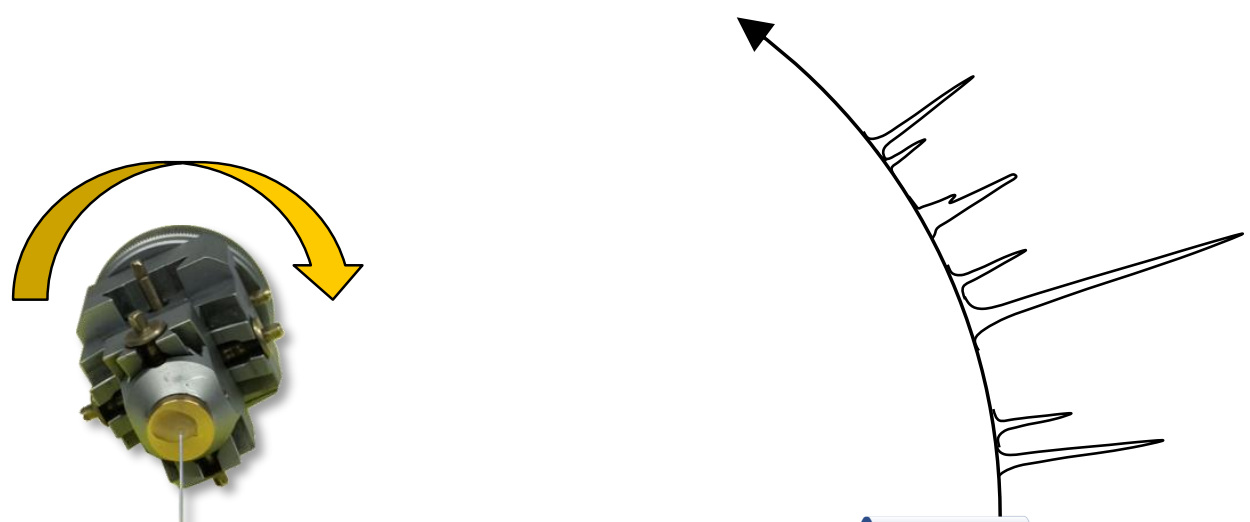


\section{Transmission (Debye-Scherrer)}

Modern 'stripe' detectors, covering with $10^{4}$ single counters a large angular range, have not improved Acquisition time the acquisition time correspondingly, but rather allowed for an even better signal/noise (counting statistics), or for time-resolved experiments with a still high angular resolution.

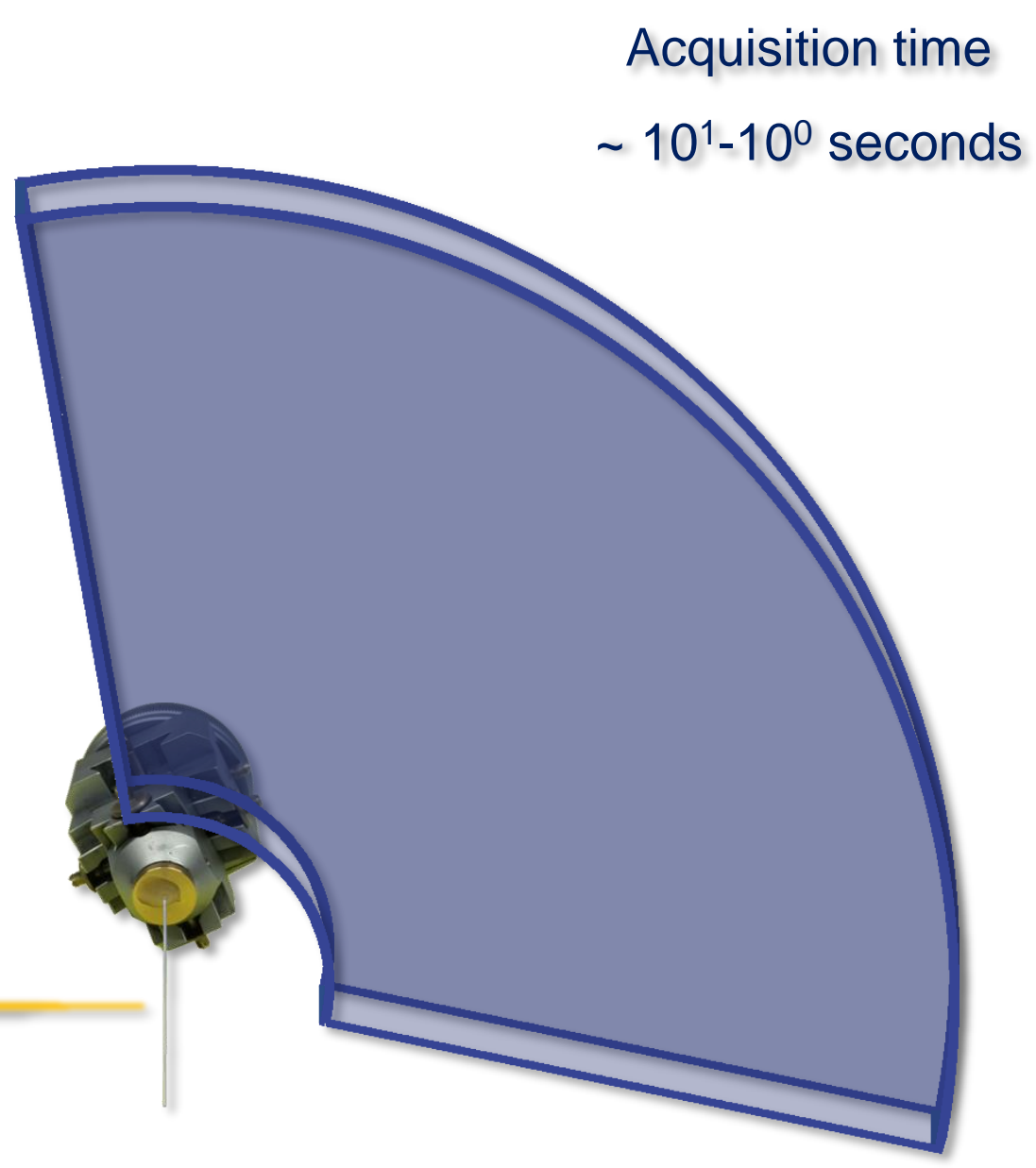




\section{Parallel data acquisition (2D)}

\section{Transmission (Debye-Scherrer)}

Area detectors offer a further advantage as they allow for integration of the full cone of diffraction, further increasing the signal/noise and/or decreasing the acquisition time possible.

These detectors are now capable of acquisition times of several 10 s of $\mathrm{kHz}$, running in continuous or pulsed mode.

Parallel data acquisition has made highthroughput presently possible, with sample delivery being presently the bottleneck

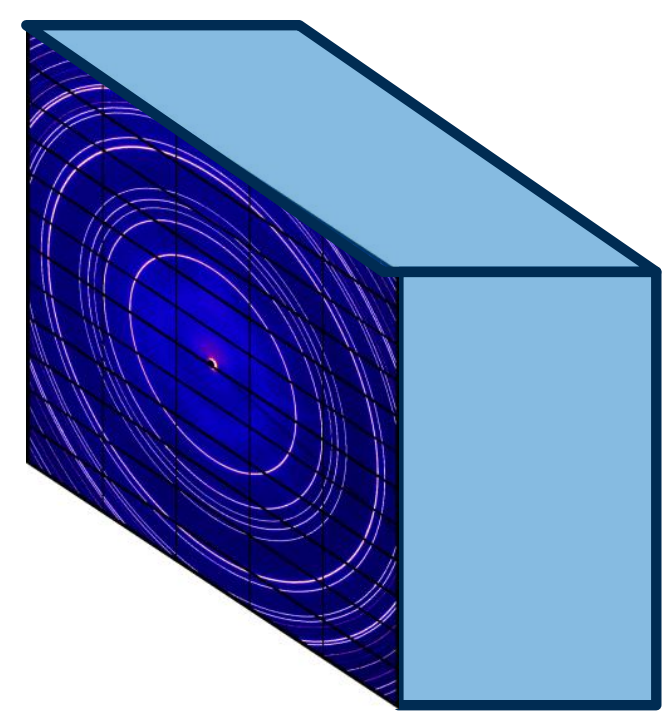


A robotic arm to automatically change samples is present in several beamlines around the word, equipped initially with multi crystal analyzers (MACs in the picture below) and with 1D 'stripe' detectors (PSD below).

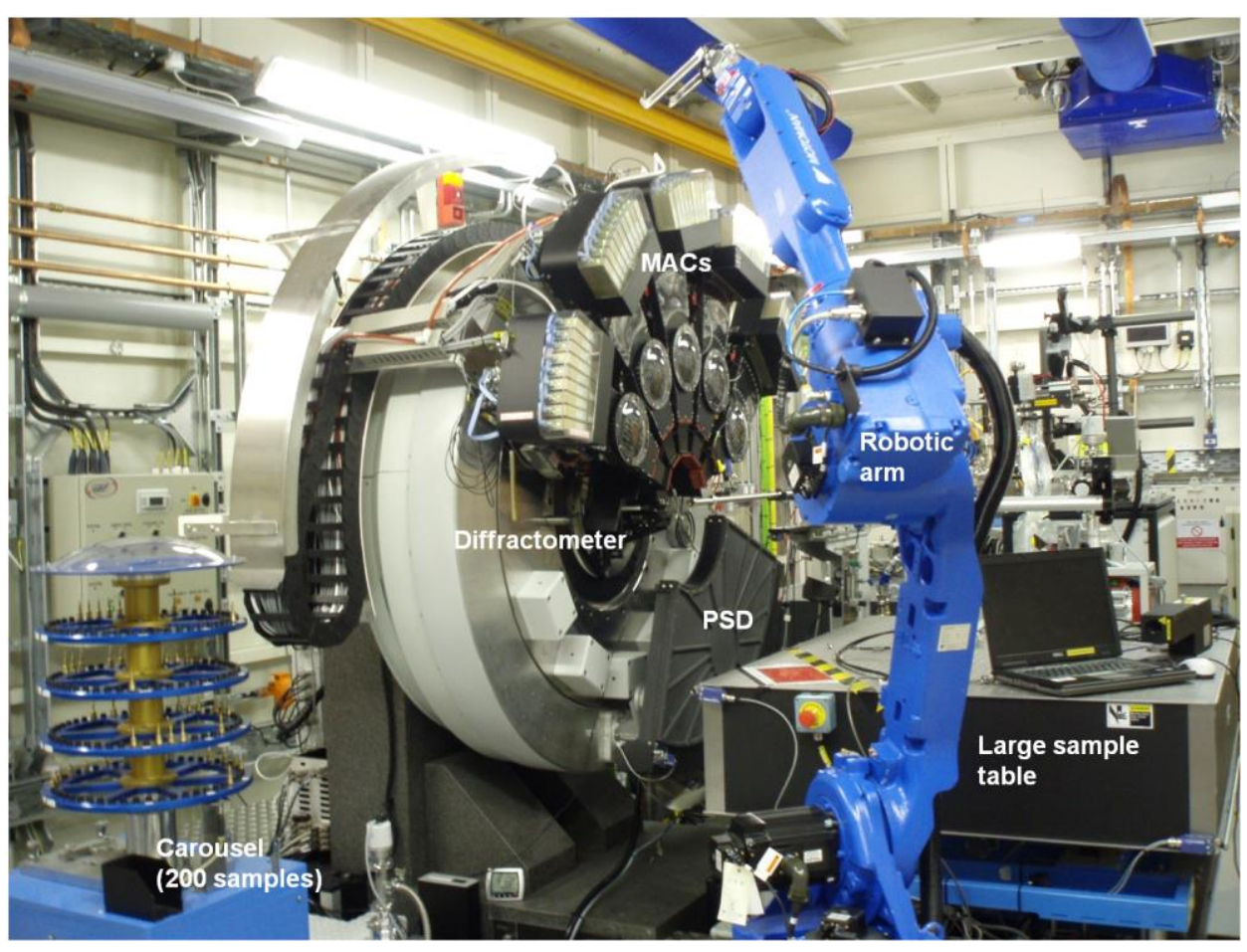

https://www.diamond.ac.uk/Instruments/Crystallography/I11.html

\section{Sample changing robot:}

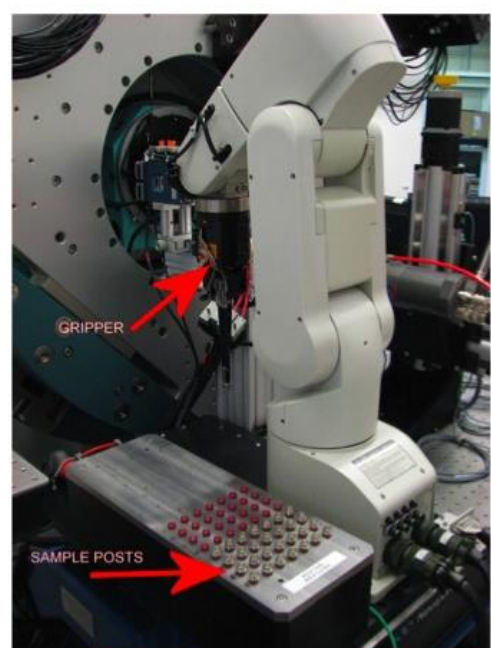

Capacity: 152 samples (56 individual and 96 in magazines)

Small footprint allowing easy access to sample area

Integrated with Cryostream 700+ for combined use of robotic operation with temperature control

Proven reliability of industrial Mitsubishi robot Locally designed Robot-Human Safety System (RHSS) to ensure safe operation

https://11bm.xray.aps.anl.gov/images/RobotDetail_large.jpg

Typically hundreds of samples can be loaded in a dedicated holder.

Acquisition times can be as low as $100 \mathrm{~s}$ 
The challenge given by capillary samples for 1D detector is that a small offset of the capillary axis with respect to the rotation axis (spinning is necessary for particle statistics) leads to an apparent peak broadening.
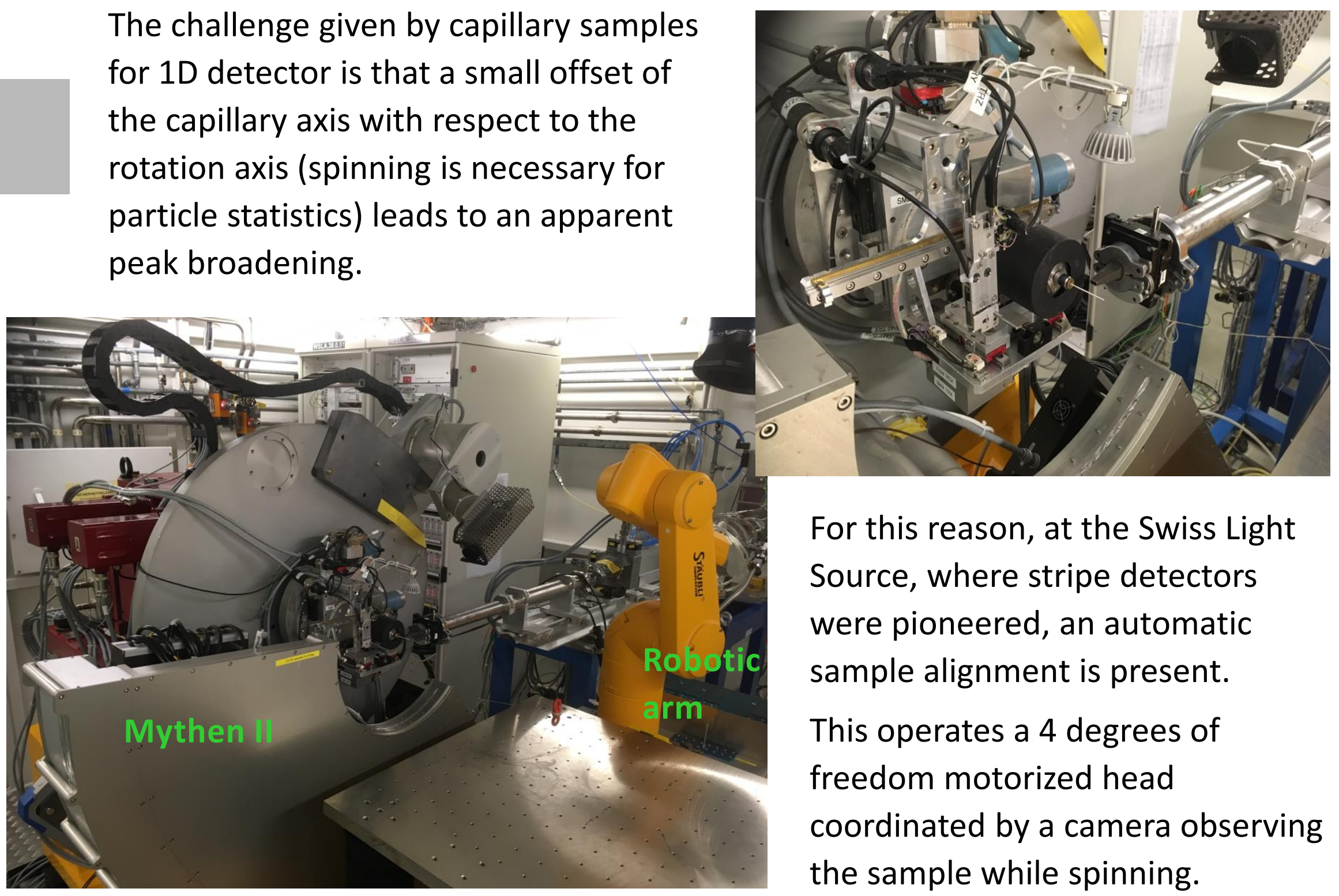

For this reason, at the Swiss Light Source, where stripe detectors were pioneered, an automatic sample alignment is present.

This operates a 4 degrees of freedom motorized head coordinated by a camera observing the sample while spinning. 
Spinning is necessary

especially with 1D detectors, as particle statistics may dominate counting statistics. In this section of a 2D image it is clear that positioning a 1D detector along the red or green arrows would not produce the same intensities.

Better result than a simple radial integration are obtained with an azimuthal one, here following the yellow line. This approach is better but not satisfactory, still.

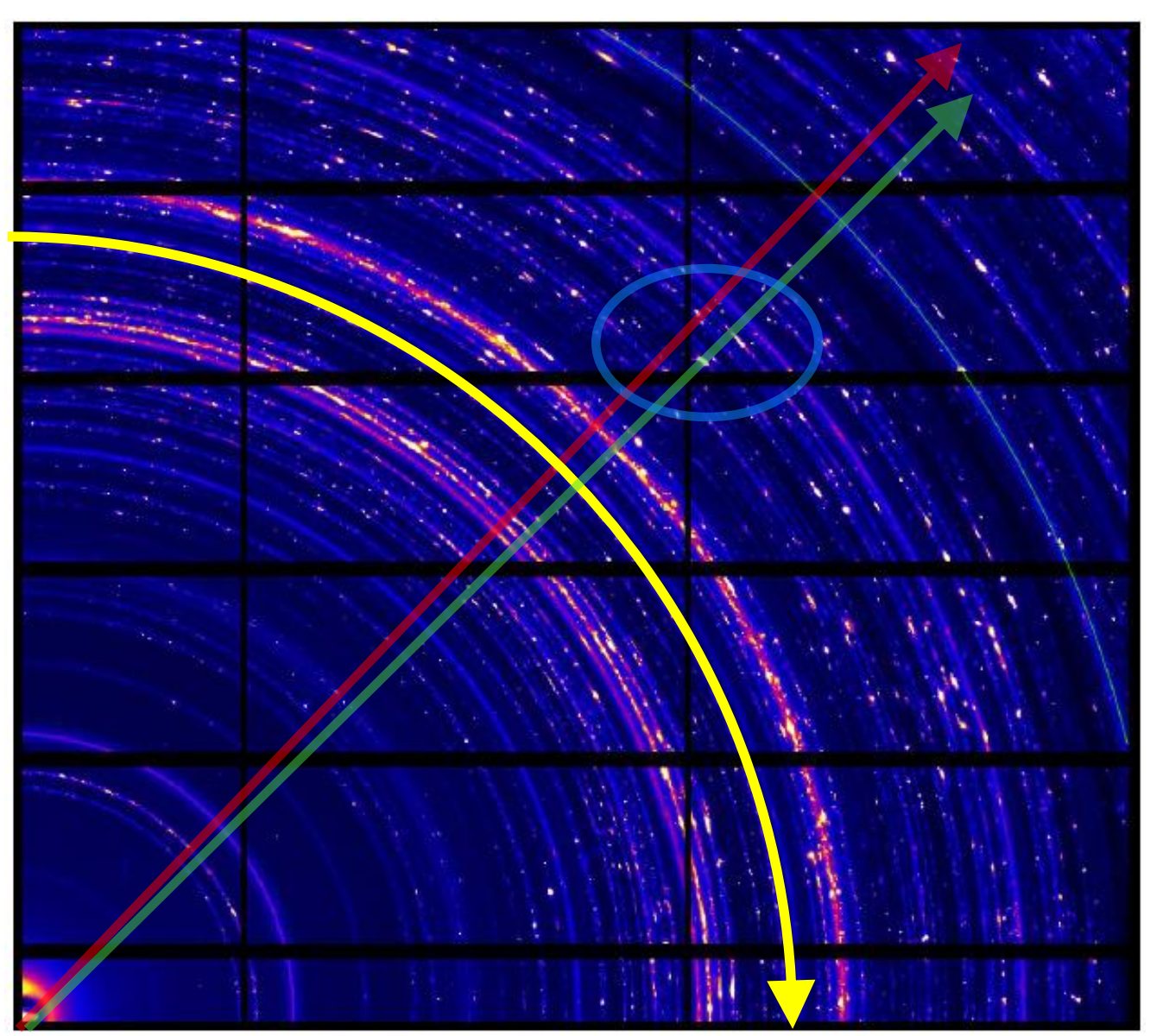


Static samples are easy to deliver to an area detector, For example in the form of a plate with several holes and X-ray transparent windows. This is the case for example of commercially available solutions for monitoring protein crystallization, adapted today to the general chemical environment.

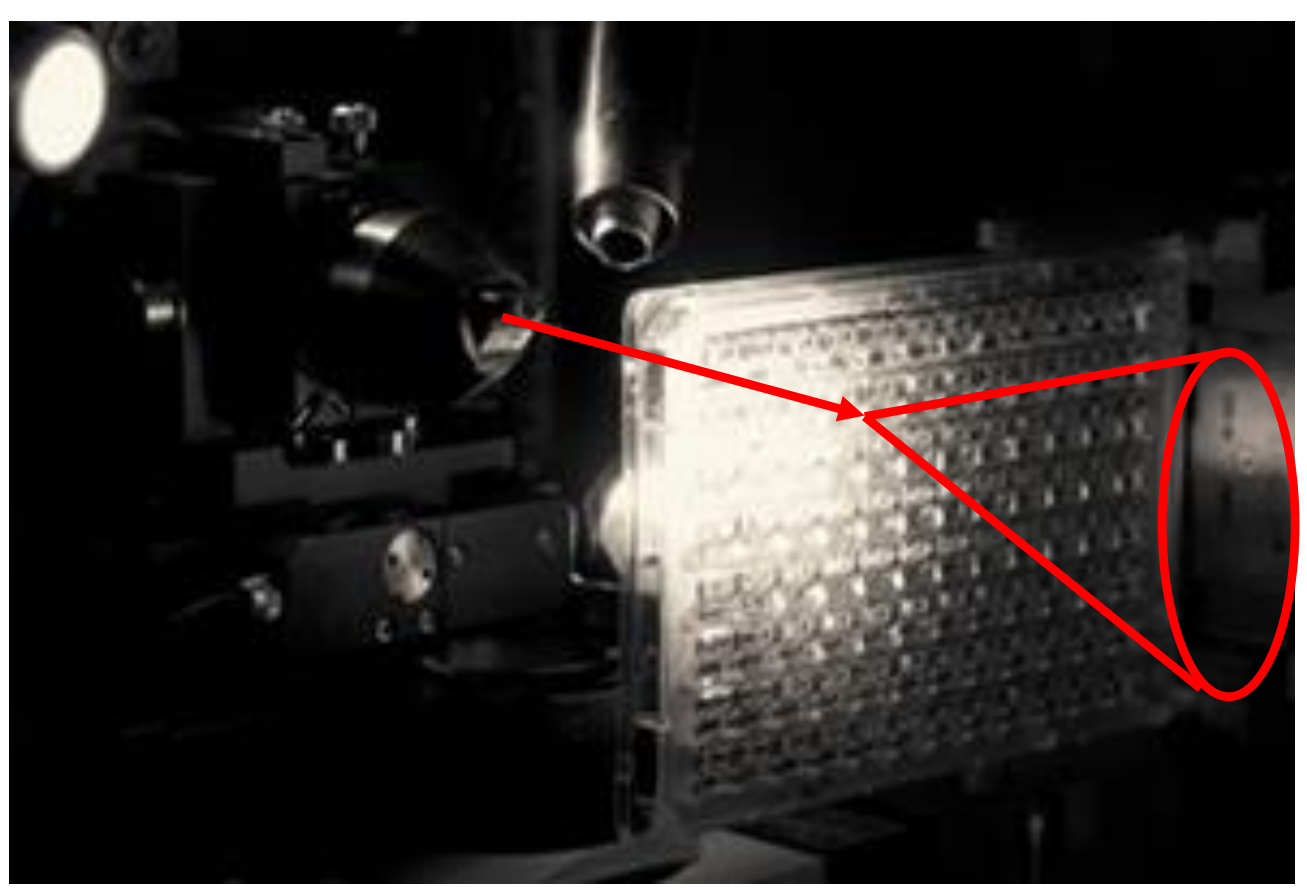

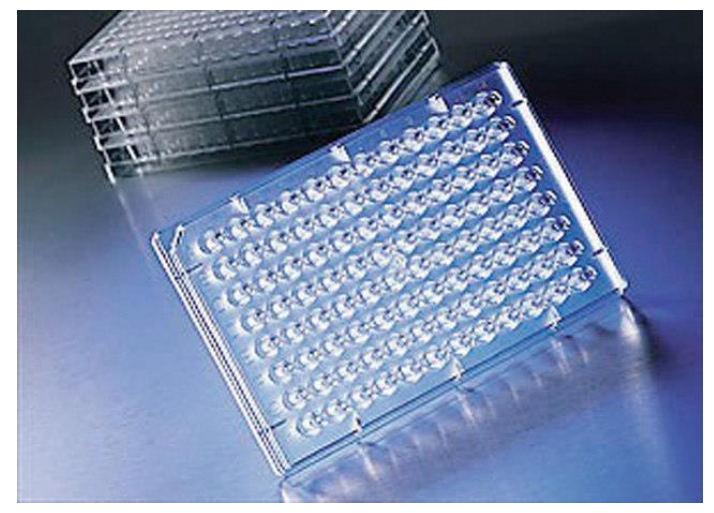

From Sigma-Aldrich

X-rays can penetrate through them, interact with the sample and be observed in transmission by a $2 \mathrm{D}$ detector positioned on the other side of the plate. Including sample change $\sim 10$ seconds per sample are possible in this way. 
Moving the sample, anyway, is mandatory for high-quality results. A more limited movement applicable to a full plate is translation, a further one being rotation at the sample position. Such an approach does not produce the best quality but a significant and simple improvement, perfectly acceptable in many cases.

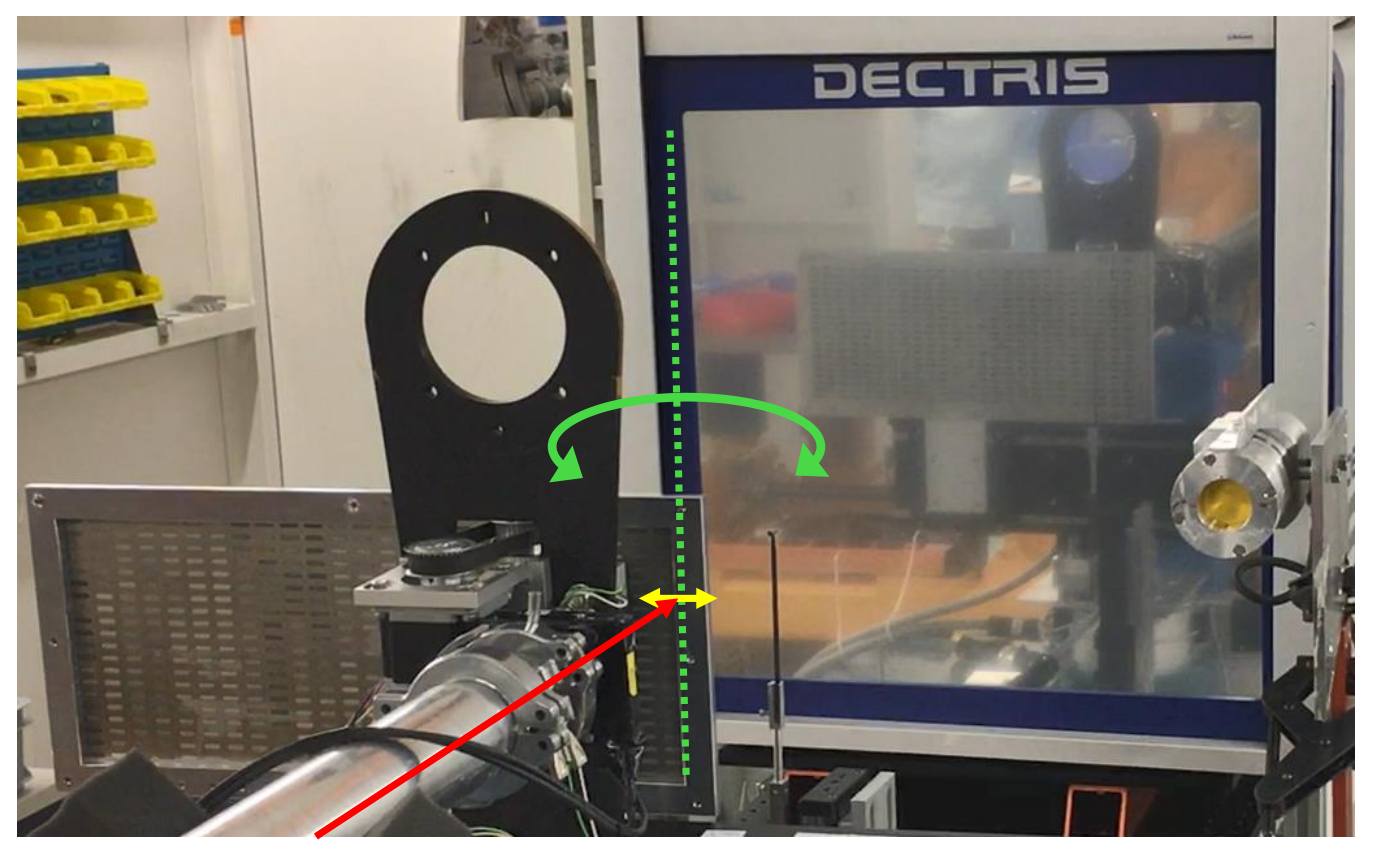

In our case a small $\pm 3^{\circ}$ rotation on the sample (green) axis was coupled with a $\pm 8 \mathrm{~mm}$ horizontal translation (yellow). Each plate contained 500 samples, time per sample is below 13 seconds including plate changing. 


\section{Plates + rotation + translation}

While motors can be accelerated it is clear such an approach is a compromise between simplicity, quality and time constraints. On the other hand results are meaningful even in the case of a non-ground sample.

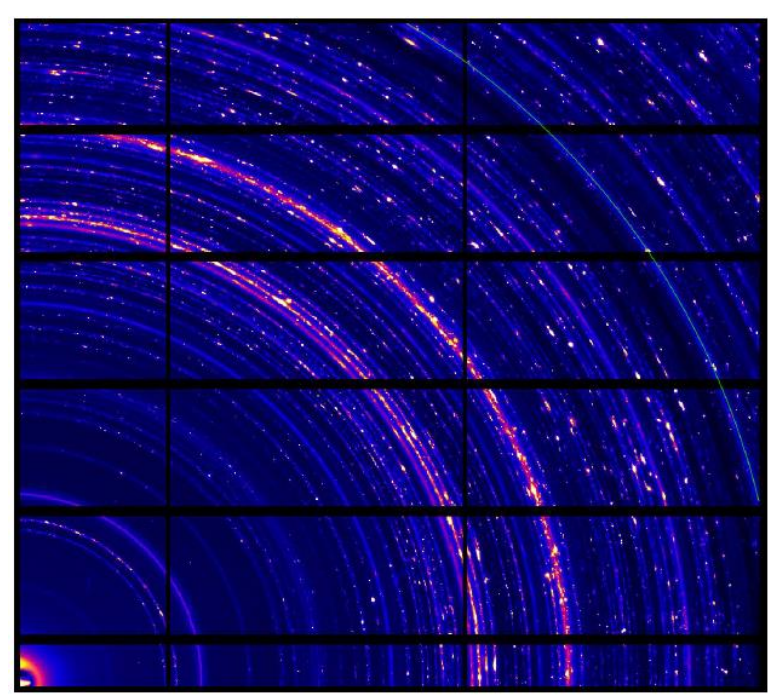

Static sample

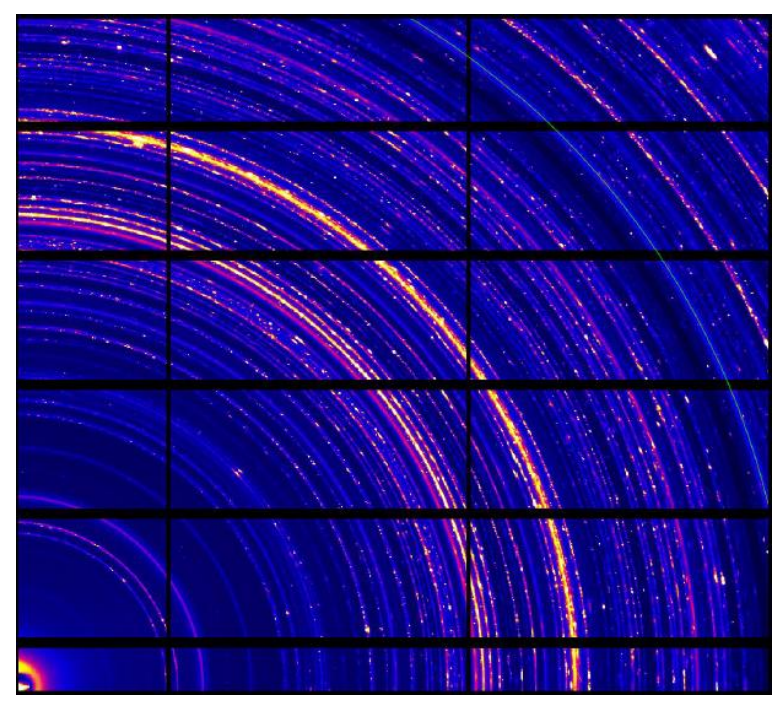

Rotation + Translation

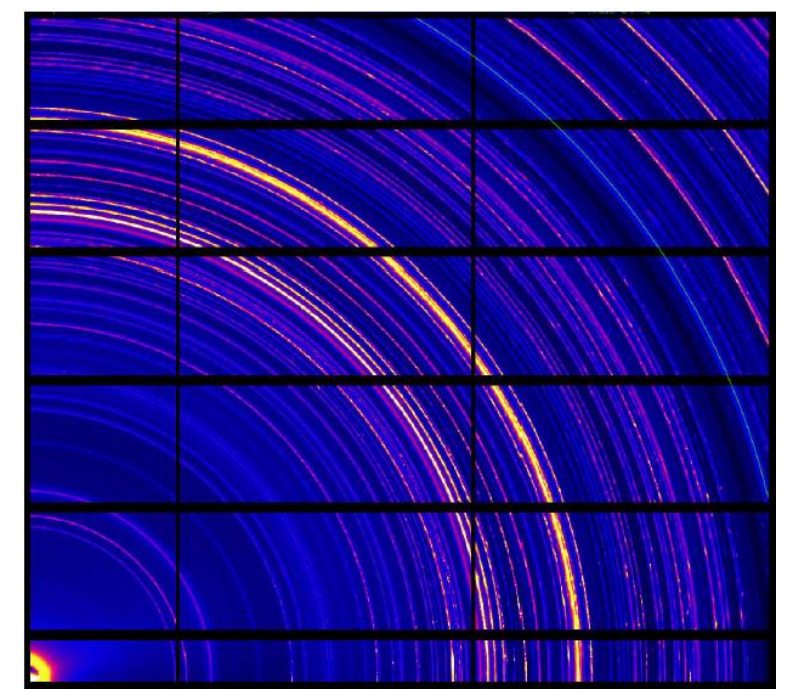

Ideal

Different ways of moving samples are a bit more complex, but not impossible. 


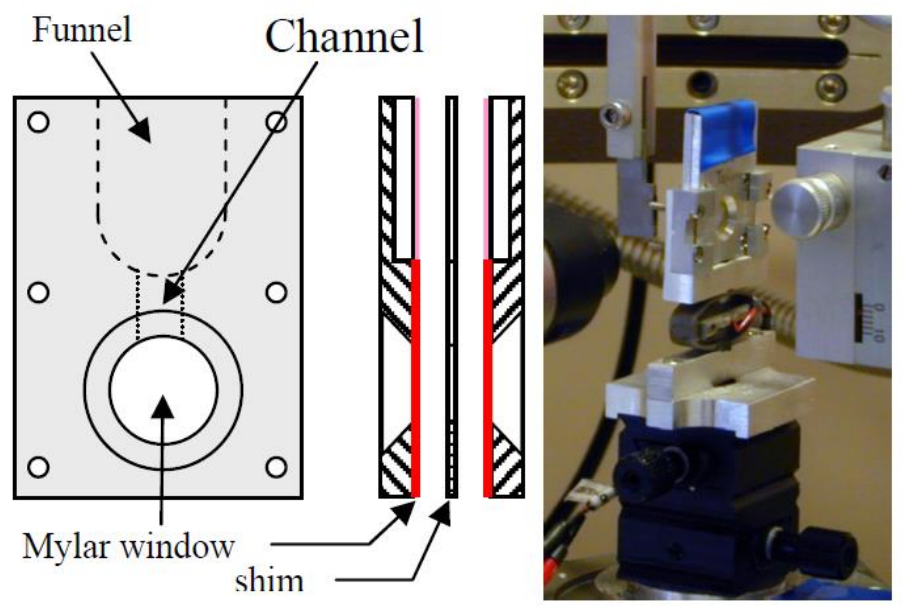

Images from P.C. Sarrazin et al., Vibrating sample holder for XRD analysis with minimal sample preparation,

International Centre of Diffration Data 2005, 48, 156-164

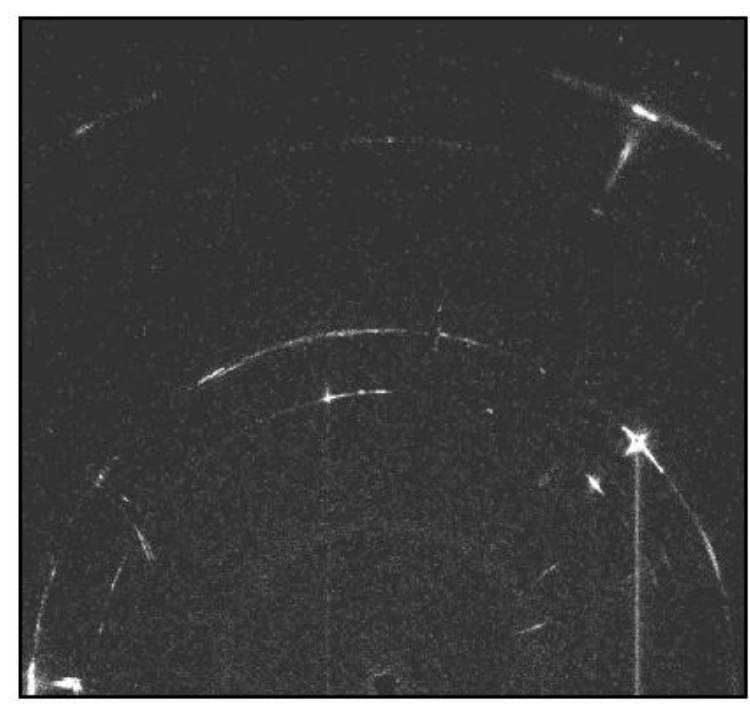

High frequencies induce a fluxional motion in the samples, which reorient particles in all possible ways. This approach was successfully pioneered in the CheMin instrument of the Mars Curiosity Rover.

It is important to note that the sample does not require any preparation, i.e. unground powders can be used.

However, vibrating large plates is not an option, given the high energy that would be required. Single samples have to be quickly exchanged. 
A similar approach was recently developed within our laboratory, specifically devoted to high-throughput. As piezo elements tend to be more fragile a different lower-frequency shaker was employed, allowing for more robust operations including a fast sample exchange.
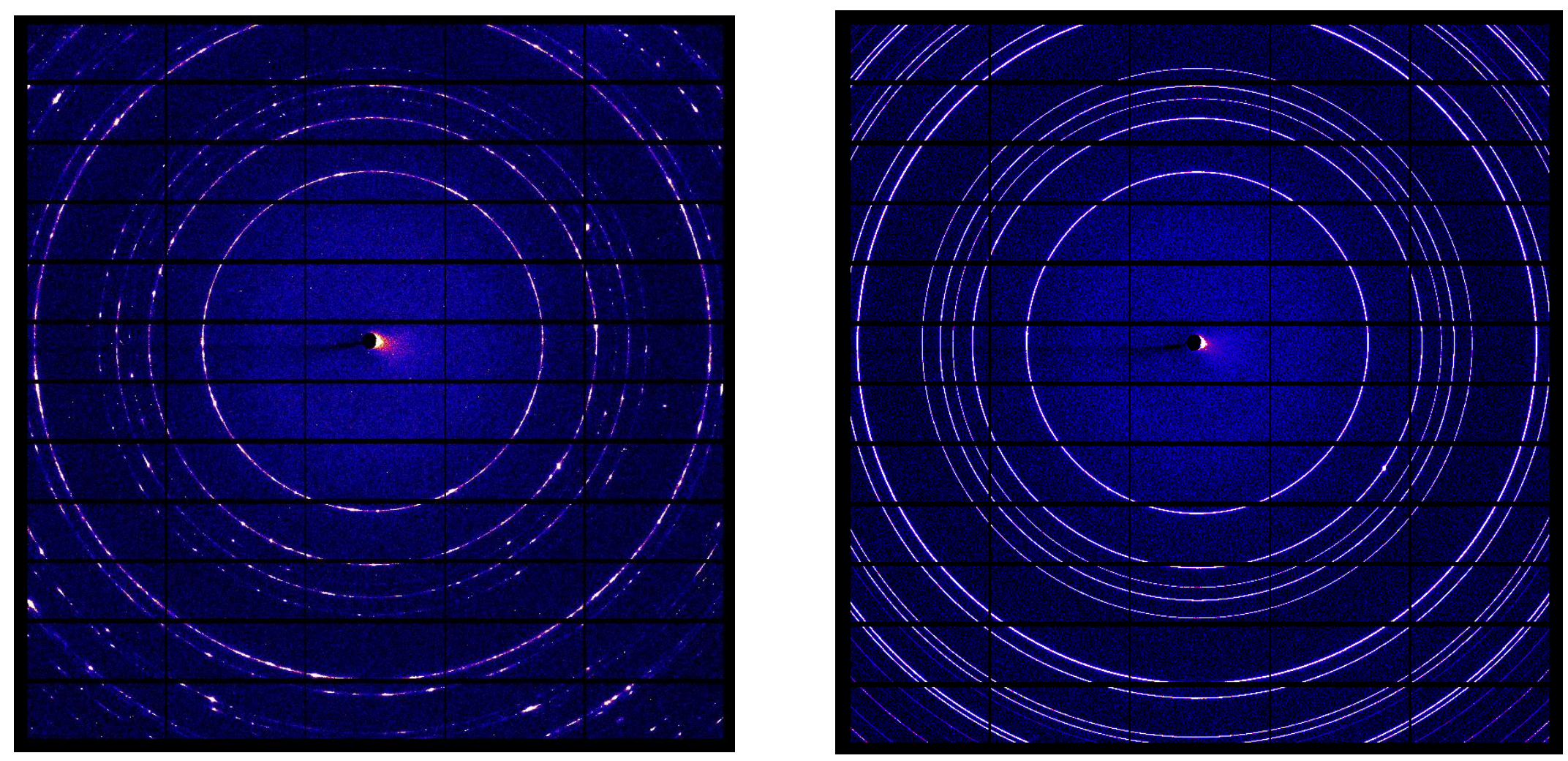


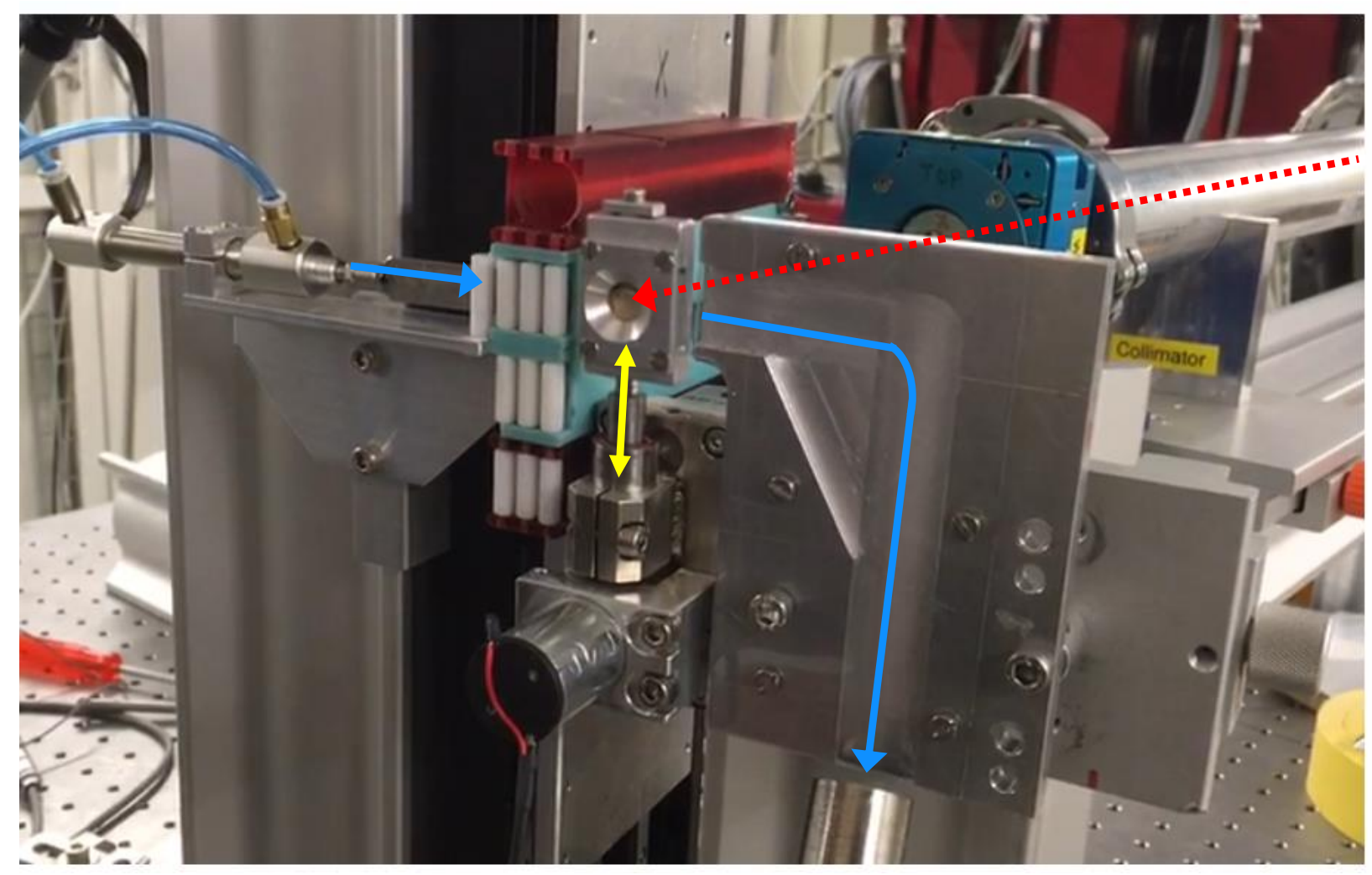

Few $s$ acquisitons High quality Automatic operation

Several washer-shaped sample holders are contained in long cartridges. Each holder can be quickly exchanged by a pneumatic drive into a vibrating seat. The sample is then vibrated and then expelled again by the arrival of a new one. 
When operations are so large clearly a full organization is needed. This was very well pointed out in Toby, B. H., et al. (2009). J. Appl. Cryst. 42, 990-993, which were anyway dealing with hundreds of samples per day

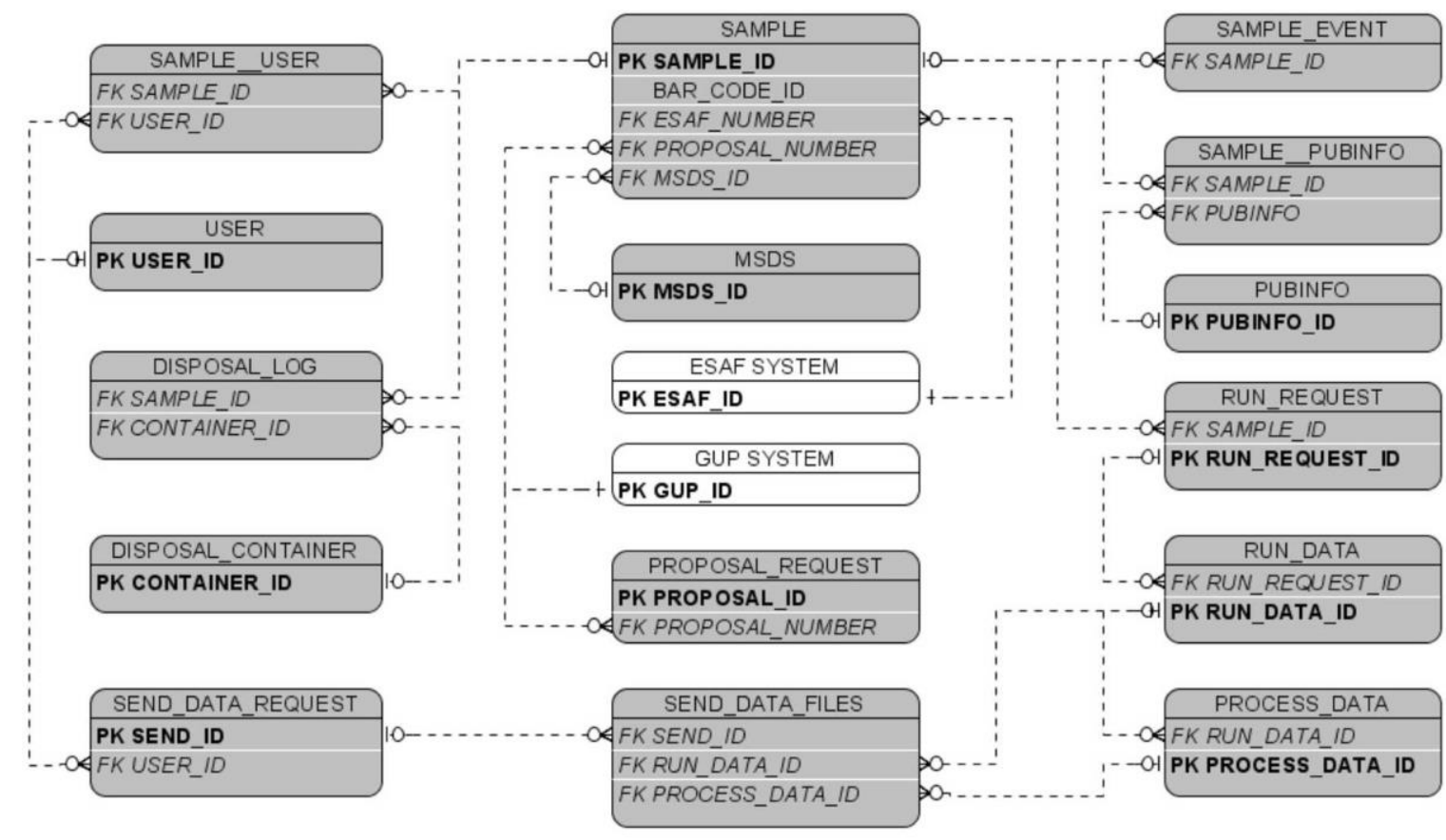

$\mathrm{PK}=$ primary key $\quad \mathrm{FK}=$ foreign key $\quad \gg 0=$ one-to-many relationship possible between entries

At 5-10 seconds per sample operations exceeding 10.000 samples per day can be accomplished, making data management even more valuable. 
Combinatorial approaches are growing, with the availability of more robotic ways of performing synthesis, sampling and every other aspect of the full scientific chain, including data analysis and interpretation.

It is worth noting that the problem was raised for us by a company commissioning the measurement of more than 50.000 samples, showing that the interest is not an academic oddity or a showcase of capability, but rather a need already present in our society.

While this remains a 'brute force' approach, it couples well with the trend to employ 'big data' and the ability to process them in novel ways.

It is also worth noting that at the number of samples that can be measured presently a synchrotron is already cheaper, per sample, than a tube-based diffractometer.

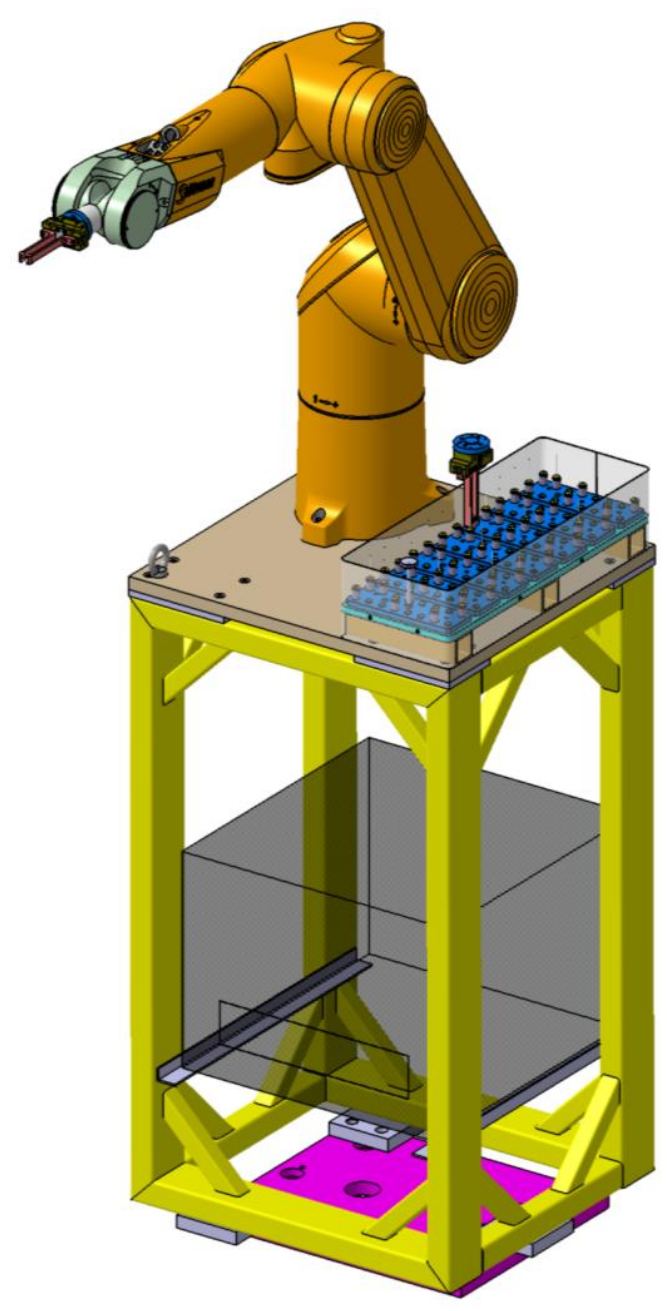




\section{Conclusions}

- Synchrotron high photon flux can be exploited for high throughput powder diffraction, on top of the more in depth studies

- Several detector geometries/quantities are available, ranging from hundreds to tens of thousands samples per day

- Fast sample delivery is possible coupled with enough movement to ensure good homogeneity even for unground samples

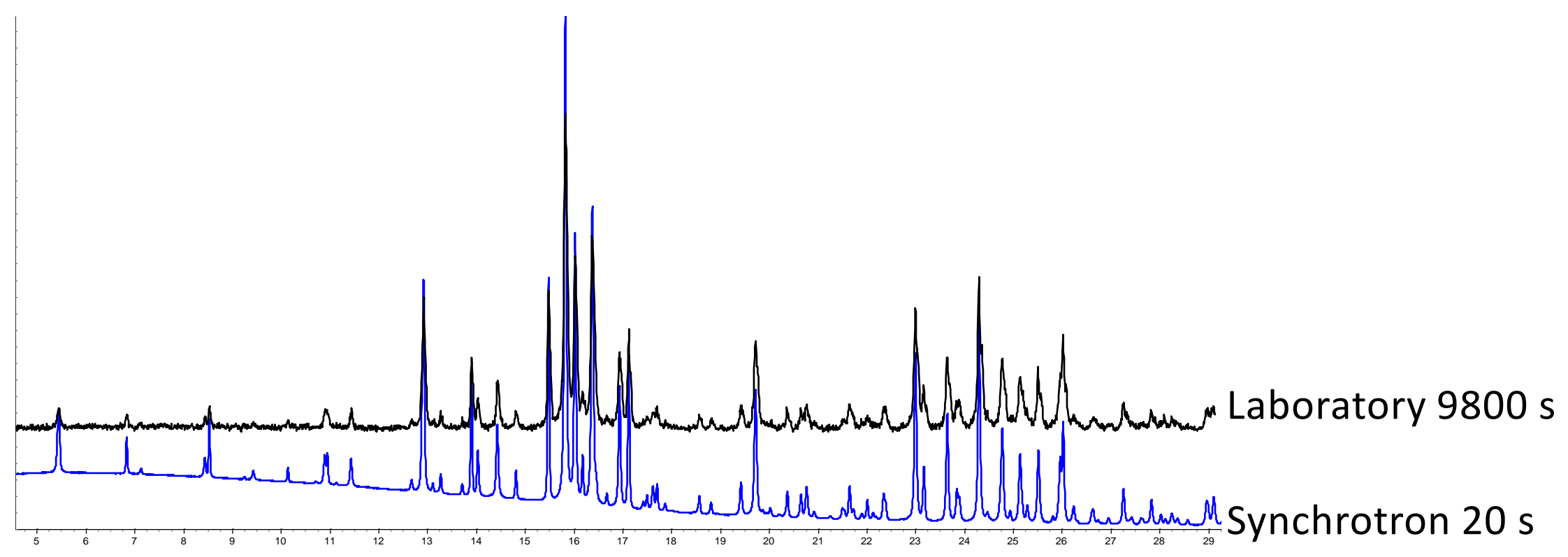

